\title{
Der Olymp und die Ochsen
}

\section{Josef E. Brandenberg}

Dr. med., Präsident der FMCH

In einem Interview im Sonntags-Blick vom 7.4.2019 stellt Carl Baudenbacher sein neues Buch Das Schweizer EU-Komplott vor und gibt Hintergrund-Informationen zum institutionellen Abkommen InstA, wie der EURahmenvertrag offiziell heisst [1]. Seine Vorschläge hätten "die Leute in Bern» abgelehnt, ohne sie ernsthaft geprüft zu haben. Niemand habe ihn angerufen. Das habe das Aussendepartement schon immer so gemacht. Die Ärzteschaft kann Herrn Baudenbacher beruhigen. Das Innendepartement macht es gleich. Interessant ist der letzte Teil des Interviews. Er berate Firmen, Regierungen und Parlamentarier. Auch die Kommission für Wirtschaft und Abgaben (WAK) des Nationalrats habe bei ihm ein Gutachten bestellt, aber am Schluss nur 12000 Franken bezahlen wollen, was in keiner Weise seiner Leistung und Reputation ent-

\section{Résumé}

Carl Baudenbacher a œuvré en tant que juge à la Cour de justice de l'AELE pendant 23 ans. Dans un entretien avec le Blick, il raconte que la Commission de l'économie et des redevances du Conseil national n'a accepté de le payer que 12000 francs pour une expertise. Pour la même tâche, une étude d'avocat zurichoise aurait réclamé 100000 francs. Un professeur de droit renommé d'une université suisse a quant à lui exigé une rémunération horaire de 650 francs pour une expertise. Dans certains cantons, les notaires sont rémunérés en fonction du montant de I'affaire. Plus la valeur d'une succession est grande au moment du partage, plus le prix d'un terrain est important lors du changement de propriétaire ou plus le capital social est conséquent au moment de la création d'une société, plus les honoraires du notaire sont élevés.

Les juristes justifient ces montants par leur réputation et leurs responsabilités. Ils ne sont soumis à aucun article de protection tarifaire, même s'ils sont spécialisés dans l'assurance sociale ou en matière fiscale.

Bien que la liberté économique selon l'article 394ss CO s'applique à la branche des assurances complémentaires, les dépassements d'honoraires médicaux sont dénoncés dans les médias et par les politiciens.

Grâce à la contribution entre autres de juristes de I'Administration, du Parlement et des juridictions, les honoraires dans le secteur des assurances sociales ne cessent de baisser. Le TARMED en vigueur fixe un tarif horaire de 119.50 francs pour une prestation médicale. Cela fait des décennies que I'on refuse la compensation du renchérissement. Sous prétexte d'accroissement du volume de prestations, on impose la neutralité des coûts, et donc la normalisation. Les valeurs quantitatives intrinsèques ont été supprimées. Quand nous libérerons-nous de cet asservissement? spreche. Eine Zürcher Anwaltskanzlei hätte für die gleiche Arbeit 100000 Franken verlangt. Baudenbacher war 23 Jahre lang Richter am EFTA-Gerichtshof. Er ist zweifellos ein Koryphäe auf seinem Gebiet. Das Gutachten lässt sich inhaltlich nicht beurteilen.

Warum wollte die WAK weniger bezahlen als ursprünglich vereinbart, wie Baudenbacher behauptet? Die sogenannten freien Berufe - Ärzte, Zahnärzte, Juristen, Treuhänder, Architekten - erbringen Dienstleistungen gemäss Obligationenrecht [2]. Die Artikel 394ff. OR regeln den "einfachen Auftrag», der getreu, sorgfältig und persönlich ausgeführt werden muss (Art. 398 OR). «Eine Vergütung ist zu leisten, wenn sie verabredet oder üblich ist» (Art. 394 Abs. 3 OR). Damit ist das eigentliche Honorar, bei Ärzten die AL, gemeint. Zudem hat «der Auftraggeber dem Beauftragten die Auslagen und Verwendungen, die dieser in richtiger Ausführung des Auftrages hatte, samt Zinsen zu ersetzen» (Art. 402 OR). Dies sind die Kanzlei-, Praxis- und externen Zusatzkosten, kurz die TL. Entweder betrachtete die WAK das Gutachten als nicht dem Art. 398 OR entsprechend oder das Honorar war unüblich. Aber was ist üblich?

Grundsätzlich sind Bewertungen und Diskussionen von Honoraren und Einkommen nur aussagekräftig, wenn der Zeitaufwand bekannt ist. Einzig Notare werden in einigen Kantonen (noch) nach der Summe des Geschäftes vergütet. Je grösser die Hinterlassenschaft bei der Erbteilung, je höher der Wert eines Grundstückes bei Handänderungen, je grösser das Aktienkapital bei Firmengründungen, umso höher ist das Notariatshonorar. Der Präsident der Luzerner Notare, selber Rechtsanwalt und Unidozent, wird wie folgt zitiert: «bei hohen Summen habe der Notar auch eine grössere Verantwortung» [3]. Im Rahmen der Gutachtertätigkeit hat der Unterzeichnende eine Erhebung über den Zeitaufwand anhand 50 Gutachten gemacht (Kausalgutachten im Sozialversicherungsbereich und FMH-Gutachten). Ergebnis: Die Anzahl Seiten - mit 440 Wörtern respektive 3000 Zeichen inklusive Leerschläge pro Seite, abzüglich der Titel- und der letzten Seite - entspricht dem Aufwand in Stunden für Aktenstudium, Sichtung des Bildmaterials, Literaturrecherche und Diktat des Gutachtens. Nach dieser Berechnung hätte die WAK für das 48-seitige Gutachten einen Stundenansatz von 261 Franken angeboten, etwas mehr als für ein FMH-Gutachten üblich ist, aber deutlich weniger als die 650 Franken pro 
Stunde, die kürzlich ein renommierter Rechtsprofessor einer Schweizer Universität für ein Gutachten verlangte. Mit diesem Stundenansatz hätte Herr Baudenbacher 30000 Franken verdient, aber immer noch ein Drittel weniger als der oben genannte Betrag von 100000 Franken einer Anwaltskanzlei in Zürich.

Dreissig Jahre vor Inkrafttreten des Obligationenrechts hat der Landarzt im Zürcher Unterland Wilhelm Meyer-Frey (1830-1906) in seinen Memoiren die damalige ärztliche Rechnungsstellung beschrieben (Zitat): «Man beginne mit einer möglichst niedrigen Einheitstaxe für Arzneien, Besuche und Operationen und erhöhe dieselbe allmählich bis sich Klagen darüber hören lassen ... Dass man seine Forderungen nicht zu hoch stellt dafür sorgen die Konkurrenten und das Publikum. Verlangt man aber zu wenig und leistet dabei doch etwas Tüchtiges, so wird die Geschäftslast unerträglich und an die Stelle der wissenschaftlichen Gründlichkeit und technischen Sorgfalt tritt die blosse Routine, das Blendwerk des Scharlatanismus» [4].

Seither sind 130 Jahre vergangen. Mit der Einführung der Sozialversicherungen - 1902 Militärversicherung, 1911 KUVG - ist der freie Arztberuf im stetigen Rückgang. Tarife bestimmen die Honorierung ärztlicher Leistungen. Das Ringen um die angemessene Abgeltung der ärztlichen Leistungen im "Armenarzt-Tarif», wie der Sozialtarif bis in die 1950er Jahre genannt wurde, zieht sich durch die ganze Geschichte der Sozialversicherungen [5]. Im aktuellen TARMED ergibt das Referenzeinkommen von 229397 Franken geteilt durch die Jahresarbeitszeit von 1920 Stunden einen Stundenansatz von 119.50 Franken [6]. Hinzu kommen die technischen Leistungen für die Praxiskosten, die nach eigenen Berechnungen rund 75\% der gesamten Einnahmen betragen, was in der Summe immer noch deutlich unter den 261 Franken liegt, die Herr Baudenbacher als zu wenig bezeichnet. Von 650 Franken pro Stunde oder gar dem Dreifachen kann die Ärzteschaft nur träumen.

Die Arzthonorare im Bereich der Sozialversicherungen seien halt durch Prämien und Steuern finanziert, wird argumentiert. Dies verlange staatliche Aufsicht und erlaube Tarifeingriffe durch den Bundesrat. Und die Rechtsgutachten im Auftrag des Bundes oder - im konkreten Fall - der Kommission für Wirtschaft und Abgaben des Nationalrates? Auch diese werden durch Steuergelder finanziert. Warum gibt es für Juristen keinen eidgenössischen Tarif mit einem Stundenansatz, berechnet aus Referenz-Einkommen und Norm-Jahresarbeitszeit? Im Bereich der Sozialversicherungen legen Gerichte die Entschädigung für die Anwaltskosten der obsiegenden Partei fest. Trotzdem bleibt das effektive Honorar Verhandlungssache zwischen Rechtsvertreter und Klient. Ein Tarifschutz à la KVG Art. 44 existiert für Anwälte nicht.
Gemäss Protokoll der Generalversammlung der Luzerner Ärztegesellschaft vom 7.12.1952 hätten «die Regierung und die Krankenkassen die Angleichung der Tarife abgelehnt, weil die Arztkosten auch ohne Teuerungsausgleich zugenommen hätten. Dass die aktivere Art des ärztlichen Vorgehens in Diagnose und Therapie eine entsprechende Mehrarbeit der Ärzte zur Folge habe, die einzelne Leistung aber dennoch nicht entsprechend dem Mass der gestiegenen Gestehungskosten honoriert werde, vermöge den Laien einfach nicht zu überzeugen" [6]. Mit dem Vorwurf der Mengenausweitung wird auch heute der Teuerungsausgleich verweigert. Würden sich die Juristen dies gefallen lassen? Die Menge der juristischen Gutachten im Auftrag des Bundes nimmt ja laufend zu.

Die Juristen sehen ihre Tätigkeit nach wie vor als freien Beruf. Ihre Reputation und Verantwortung lassen sie sich gebührend honorieren. Der Ärzteschaft wurden die quantitativen Dignitäten gestrichen. Die Ärzteschaft wird von Medien, Politik, ja vom Bundesrat persönlich an den Pranger gestellt. Selbst für Privathonorare erhält sie Prügel mit der Ethikkeule [8]. Juristenhonorare sind für Medien und Politik tabu. Für den Niedergang der Ärzteschaft sorgt unter anderem, aber massgebend der halbgöttliche Olymp, bestehend aus 47 Juristen im Nationalrat und 17 im Ständerat, aus unzähligen Juristen in der Verwaltung und an Gerichten [9].

Das lateinische Sprichwort lautet "Quod licet Jovi non licet bovi». Die Ärzteschaft hat sich im Verlaufe der Jahrzehnte tatsächlich zu Ochsen machen lassen. Laut Wikipedia [10] könne man diese "zu friedlichen, wenig aggressiven Nutztieren mit grosser Arbeitskraft abrichten ... Bereits ab Mitte des 4. Jahrtausends vor Christi sei dokumentiert, dass Ochsen unter dem Joch den Karren ziehen.» Wem die Machenschaften der Veterinärmedizin zwecks wirtschaftlicher Ausbeutung der Tiere zuwider sind, kann bos, bovis (lat.) auch geschlechtsneutral mit Rindvieh übersetzen. Die Frage bleibt die gleiche: Wann streifen wir das Joch ab und durchbrechen den Zaun?

\section{Literatur}

1 Interview mit Carl Baudenbacher, Sonntags-Blick vom 7.4.2019.

2 Schweizerisches Obligationenrecht. Dreizehnter Titel: Der Auftrag. 1911.

3 Von Däniken A. Gebühren-Monopol für Notare soll fallen. Luzerner Zeitung, 4.5.2019, S. 23.

4 Meyer AD. Ein Landarzt der Gründerzeit. Wilhelm Meyer-Frey (1830-1906) in seiner Autobiographie. Mitteilungen der Antiquarischen Gesellschaft Zürich, Band 61. Zürich: Verlag Hans Rohr; 1994. 123.

5 Eschle A. Geschichte der Ärztegesellschaft des Kantons Luzern 1811-1961. Bern: Hans Huber; 1962. 44-320.

6 Rundschreiben Nr. 2018_10_2.1 vom 1.10.2018 zuhanden der Ärztekammer vom 25.10.2018.

7 Eschle A. Geschichte der Ärztegesellschaft des Kantons Luzern 1811-1961. Bern: Hans Huber; 1962. 304

8 SRF, Kassensturz vom 16.4.2019.

9 Brandenberg JE. Halbgötter in Schwarz. Schweiz Ärzteztg 2019;100(9):294-6

10 Wikipedia. Der Ochse. 